\title{
Prescription pattern of alpha blockers in the treatment of benign prostatic hyperplasia in India: a paper based survey
}

\author{
Vinay Tomar', Bhoopat Singh Bhati², Biswajit Datta ${ }^{3}$, Laxman Bellamkonda ${ }^{4}$, \\ Hiren K. Prajapati5*, Alok Chaturvedi ${ }^{5}$, Nilanj Dave ${ }^{5}$, Amit B. Jain ${ }^{5}$
}

\author{
${ }^{1}$ Urology Research and Development Centre, SMS Medical College and Hospital, Jaipur, Rajasthan India \\ ${ }^{2}$ Department of Urology, Ruby Hall Main Clinic, Pune, Maharashtra, India \\ ${ }^{3}$ Department of Urology, North Bengal Medical College, Siliguri, West Bengal, India \\ ${ }^{4}$ Hyderabad Urology and Andrology Centre, Hyderabad, Telangana, India \\ ${ }^{5}$ Department of Medical Affairs, Intas Pharmaceuticals Limited, Ahmedabad, Gujarat, India
}

Received: 15 November 2019

Revised: 16 December 2019

Accepted: 17 December 2019

*Correspondence:

Dr. Hiren K. Prajapati,

Email: hiren_prajapati@intaspharma.com

Copyright: (C) the author(s), publisher and licensee Medip Academy. This is an open-access article distributed under the terms of the Creative Commons Attribution Non-Commercial License, which permits unrestricted non-commercial use, distribution, and reproduction in any medium, provided the original work is properly cited.

\begin{abstract}
Background: Benign prostatic hyperplasia (BPH) is the most common urological condition. The treatment of BPH depends on the severity of symptoms which aims to improve symptoms, lower the risk of progression and improve quality of life. The aim of this survey was to understand the prescription pattern of alpha blockers in the treatment of $\mathrm{BPH}$ among clinicians of India.

Methods: A cross-sectional questionnaire-based survey was conducted between September to December 2018. Data regarding the management of BPH using $\alpha$-blockers were filled by clinicians and collated for data analysis using appropriate statistical test.

Results: Total of 1764 clinicians' responses was collected and the result was analysed. According to the survey, $47.68 \%$ of clinicians felt that severity of the BPH symptoms is most common deciding factor for medical management of BPH. For the pharmacological management of BPH patients, around $58 \%$ of clinicians opted for $\alpha$ blockers monotherapy as a preferred option. Among $\alpha$ blockers, $65.14 \%$ of clinicians preferred tamsulosin as first line therapy for management of BPH patients. In this survey, $81.75 \%$ of clinicians believed that tamsulosin offers highest persistence rate among commonly prescribed $\alpha$ blockers. Looking at the switching to a second $\alpha$-blocker, $75.45 \%$ of clinicians felt that tamsulosin shows the highest return rate following initiation of a second $\alpha$-blocker. More than $90 \%$ of clinicians felt that favourable efficacy or tolerability of tamsulosin is due to its highest persistence and highest return rates.

Conclusions: Tamsulosin is the most commonly preferred and prescribed $\alpha$-blocker by Indian clinicians due to its favourable efficacy or tolerability.
\end{abstract}

Keywords: Benign prostatic hyperplasia, Lower urinary tract symptoms, $\alpha$-blocker, Tamsulosin, Persistence rate, Return rate

\section{INTRODUCTION}

Benign prostatic hyperplasia (BPH) is the most common urological problem among the aging male population with increasing prevalence throughout the world. ${ }^{1} \mathrm{BPH}$ is a complex disease from the etiological and pathogenesis point of view. ${ }^{2}$ BPH may compress the urethra which results in anatomic bladder outlet obstruction (BOO); 
BOO may present as lower urinary tract symptoms (LUTS) which may markedly affect quality of life (QoL). ${ }^{1}$

The treatment of BPH depends on the severity of symptoms. Management approaches include conservative management, medical therapy, minimally invasive, endoscopic or open surgery. ${ }^{3,4}$ Men with bothersome LUTS without complications from BPH, such as urinary retention, hydronephrosis or impaired kidney functions are often good candidates for medical therapy.

The aim of medical therapy is to improve symptoms, lower the risk of progression and improve QoL. There are many guidelines and algorithms along with different drug therapy options are available for BPH management. Different categories of medication are used for LUTS due to $\mathrm{BPH}$, including those used to reduce $\mathrm{BOO}$ to treat bladder overactivity. ${ }^{6}$

The evolution of alpha blocker therapy for BPH has focused mainly on improving convenience and tolerability. ${ }^{7}$ Alpha blockers are the most effective, least costly, and better tolerated drugs used for relieving signs and symptoms of $\mathrm{BPH}^{7}$ Five long-acting alpha 1 blockers are approved by the US Food and Drug Administration for treatment of symptomatic LUTS or BPH namely, terazosin, doxazosin, tamsulosin, alfuzosin and silodosin. ${ }^{7}$ All are well tolerated and have comparable dose-dependent effectiveness. In India, clinically four alpha 1 blockers are used namely, terazosin, tamsulosin, alfuzosin and silodosin. The analysis of $\alpha$-blocker prescription patterns is important to assess medication adherence, adverse effects, effectiveness, and outcomes. ${ }^{7}$

\section{Objective}

The aim of this survey was to understand the prescription pattern of alpha blockers in the treatment of BPH by clinicians of India.

\section{METHODS}

A cross-sectional questionnaire-based survey was conducted among Indian clinicians. The duration of this survey was from September 2018 to December 2018. The clinicians practicing independently and in the corporate hospitals in 66 cities across 15 states of India like Gujarat, Maharashtra, Uttar Pradesh, Odisha, Karnataka, Tripura, Uttarakhand, Andhra Pradesh, Madhya Pradesh, Rajasthan, Kerala, Tamil Nadu, Bihar, Jharkhand and Telangana as well as Union territories of India like Goa and Delhi were included in this survey. A paper-based questionnaire survey (Figure 1) was provided to all study participants. The overall information regarding the approach to the management of BPH patients and usage of alpha blockers in these patients was collected. The filled survey forms were returned by the clinicians to the medical representatives. Number of responses to each question was categorized and percentages for all the responses were calculated using outlook excel formula. Missing data was also considered for result analysis.

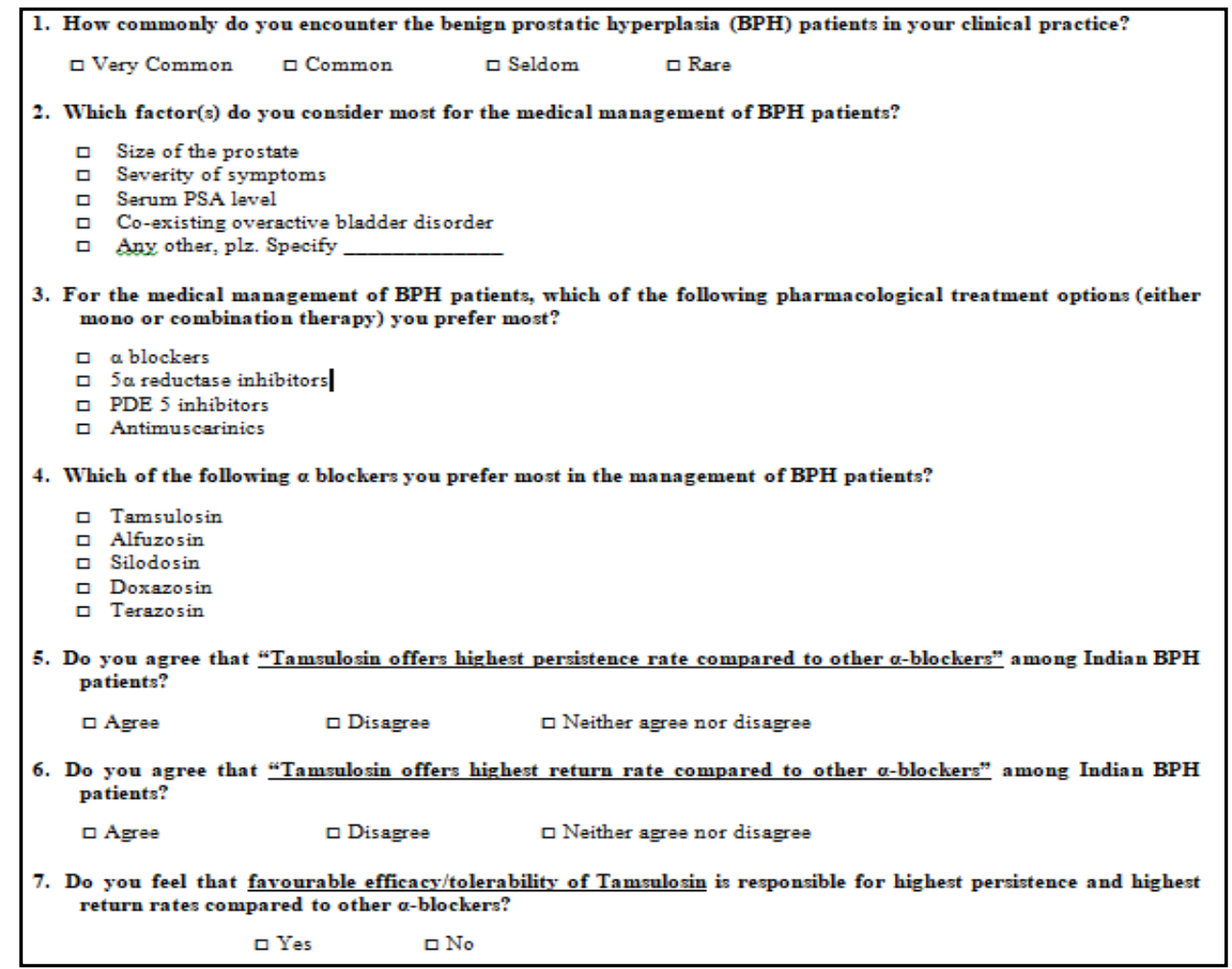

Figure 1. Survey questionnaires tool. 


\section{RESULTS}

Total 1764 clinicians participated in this survey. The results of the survey were subjective based on the clinicians' clinical experience and practice in India. In our survey, about $95 \%$ (1665 of 1764) of clinicians believed that the prevalence of BPH is very common/common in our country. This is reflecting a significant public health problem of BPH among Indians (Figure 2).

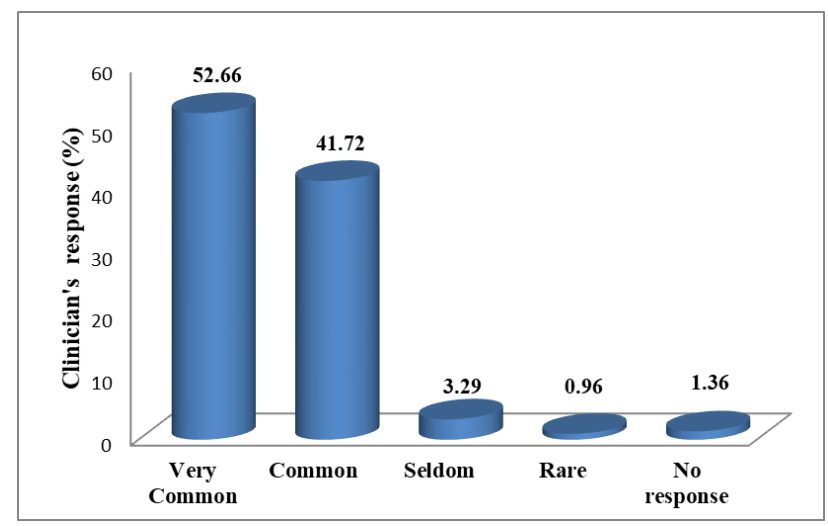

Figure 2: Frequency of BPH patients encountered in clinical practice.

Several factors should be considered while managing these patients. According to survey, 47.68\% (841/1764) of the clinicians believed that severity of the BPH symptoms is the most common deciding factor for medical management of BPH patients (Figure 3) followed by size of the prostate $(10.26 \%)$.

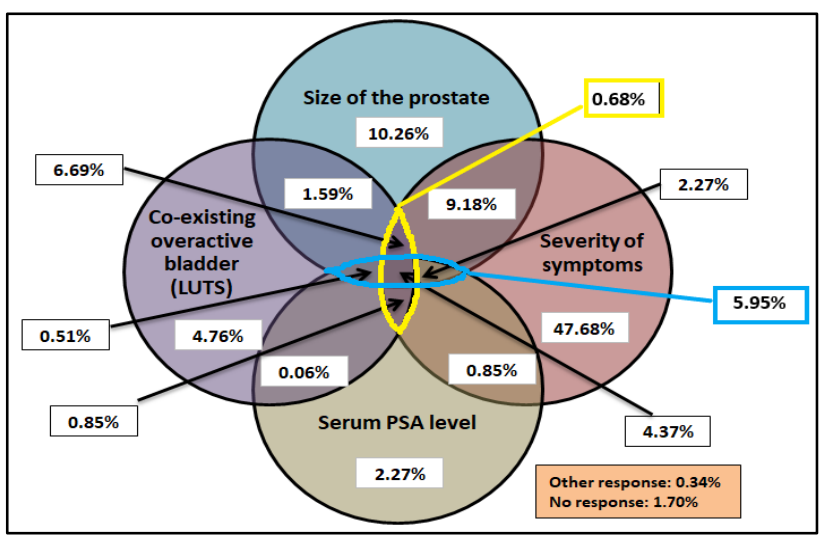

Figure 3: Clinical factors considered during BPH management.

Among the available pharmacological management options for BPH, more than 58\% (1025/1764) clinicians favoured $\alpha$ blockers monotherapy as a preferred option followed by combination of $\alpha$ blockers and $5 \alpha$ reductase inhibitors (24.15\%) (Figure 4).

Among the $\alpha$ blockers, $65.14 \%$ (1149 of 1764) clinicians preferred tamsulosin as first line therapy for management of BPH patients (Figure 5).

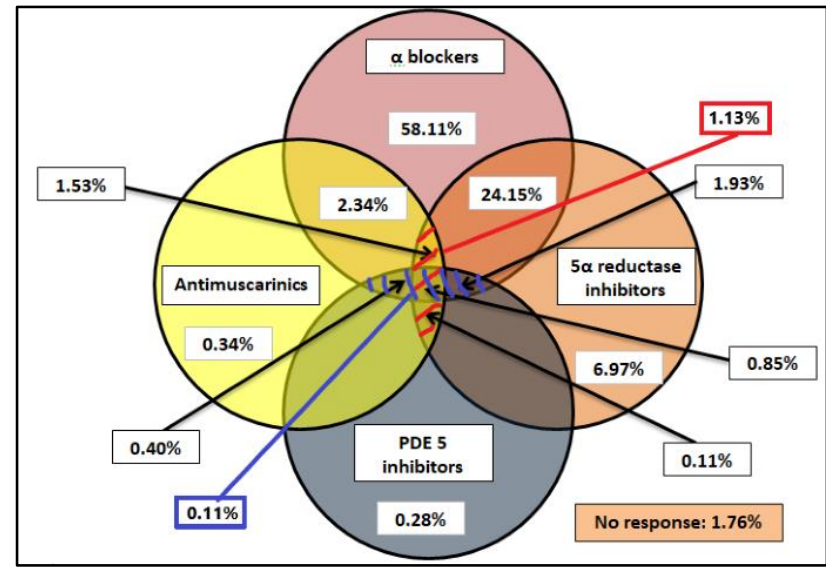

Figure 4: Preferred class of drugs for BPH management.

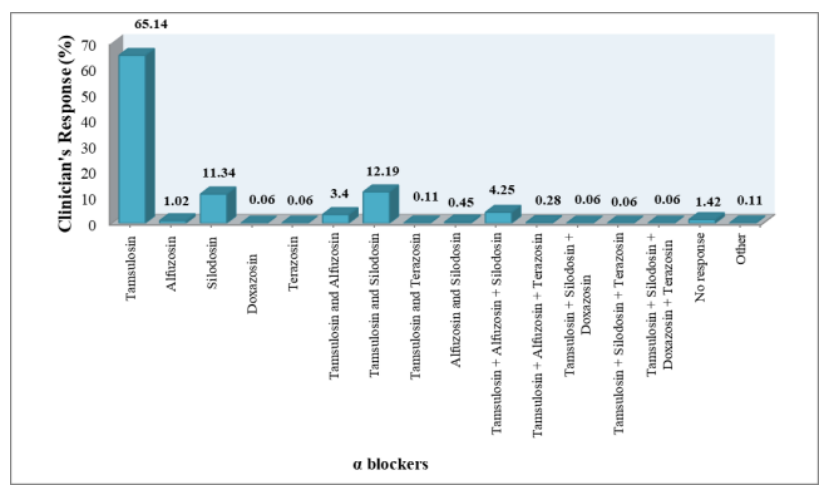

Figure 5: Preferred $\alpha$ blockers for BPH management.

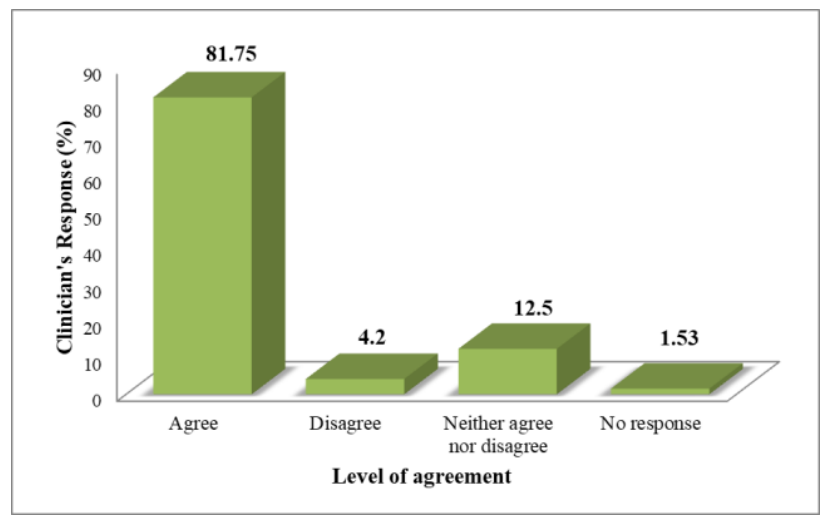

Figure 6: Level of agreement of clinicians on highest persistence rate with tamsulosin compared with other a-blockers.

Around $81.75 \%$ (1442/1764) of clinicians believed that tamsulosin offers highest persistence rate compared to other $\alpha$ blockers (Figure 6).

Among patients who switched to a second $\alpha$-blocker, $75.45 \%$ (1331 of 1764) of clinicians felt that tamsulosin shows the highest return rate following initiation of a second $\alpha$-blocker when compared with other $\alpha$-blockers (Figure 7). 


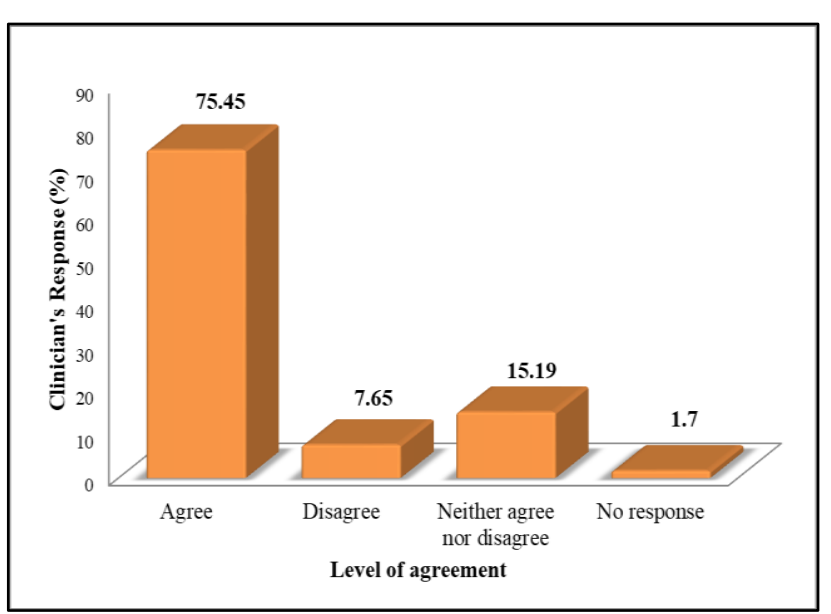

Figure 7: Level of agreement of clinicians on highest return rate with tamsulosin compared with other $\alpha$ blockers.

Based on the Figure 6 and 7 data, it can be concluded that tamsulosin offers better efficacy, safety and tolerability for the BPH management. This has been reflected in survey, where more than $90 \%$ (1595 of 1764) of clinicians believed that favourable efficacy or tolerability of tamsulosin is responsible for highest persistence and highest return rates compared to other $\alpha$-blockers (Figure 8).

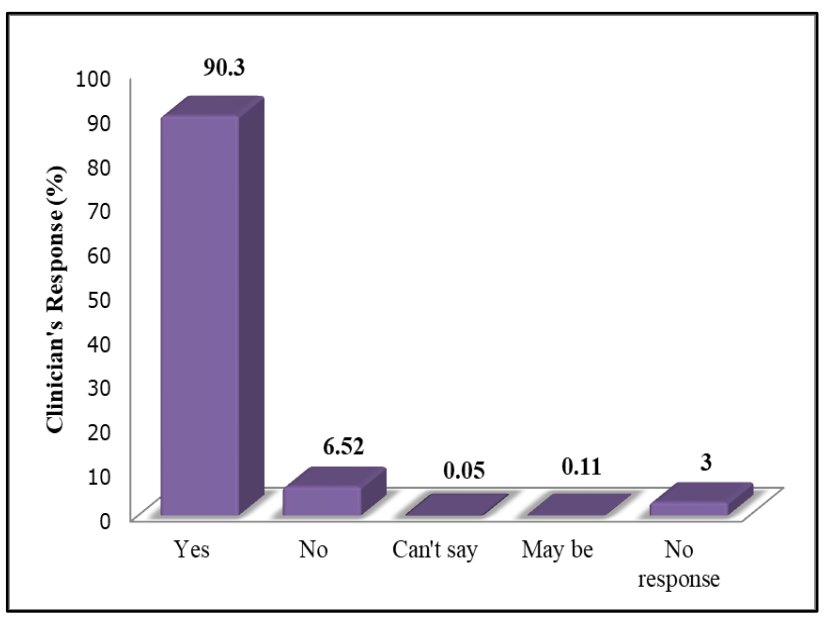

Figure 8: Clinician's response on favourable efficacy or tolerability of tamsulosin.

\section{DISCUSSION}

$\mathrm{BPH}$ is also called an old man's disease. Due to increase in life expectancy, the number of elder patients with BPH is also increasing rapidly. ${ }^{8}$ Similarly, in our survey, over $95 \%$ of clinicians believed that cases of BPH are very common or common in India. BPH is chronic progressive disease which is associated with serious long-term complications, such as acute urinary retention and hydronephrosis. Patients with $\mathrm{BPH}$ experience a significant deterioration in QoL due to severity of symptoms. ${ }^{9}$ Among available factors, the severity of symptoms was most common deciding factor for the management of BPH patients in our survey. This result was similar to prostate research on behaviour and education (PROBE) survey conducted by Emberton et al. ${ }^{10}$ In contrast to our result, size of the prostate was most common factor governing the combination therapy for medical management of $\mathrm{BPH}$ patients in a study conducted by Sabnis et al. ${ }^{11}$ Our study shows that $58.11 \%$ clinicians preferred $\alpha$-blockers as first choice treatment while $24.15 \%$ clinicians prescribed 5 $\alpha$-reductase inhibitors in combination with $\alpha$-blocker. This result was similar to a study conducted by Sabnis et al. ${ }^{11}$ This is consistent with the current clinical guidelines as well as practice recommendations in most countries. ${ }^{12-14}$ Thus, the current management is appropriately focused on a rapid relief of symptoms. Among the $\alpha$-blockers, tamsulosin was the most commonly prescribed $\alpha$-blocker in our study. This result was similar to study conducted by Sabnis et al. ${ }^{11}$ This may be attributable to the older and most trusted drug, lower cost as well as easy availability of tamsulosin across the country. In our survey, around $82 \%$ of clinicians believed that tamsulosin offers "highest persistence rate" among $\alpha$-blockers and around $75 \%$ of clinicians believed that tamsulosin offers "highest return rate" following initiation of a second $\alpha$-blocker when compared with other $\alpha$-blockers. Our findings were similar to a study conducted by Moon et al. ${ }^{15}$ This reflects the patient satisfaction with use of tamsulosin in BPH management. In our survey, more than $90 \%$ of clinicians believed that favourable efficacy/tolerability ratio of tamsulosin is responsible for this highest persistence and return rates compared with other $\alpha$-blockers.

The study has some limitations. First, the responses collected were subjective and may have so called possibility of response bias. Secondly, it did not include detailed clinical information such as prostate volume, symptom score, uroflowmetry, or residual volume, which may influence the choice and response to medication. Third, the study design was close ended questionnaires which may have impact on the final results. Despite these limitations, this survey helps clinicians to choose most appropriate $\alpha$-blocker in their real clinical practice for the management of BPH patients.

\section{CONCLUSION}

The burden of BPH is high among Indian population. Most of the Indian clinicians preferred $\alpha$-blockers as first choice treatment for $\mathrm{BPH}$ management either as monotherapy or combination with $5 \alpha$-reductase inhibitors. Among the $\alpha$-blockers, tamsulosin was most commonly prescribed by clinicians due to its favourable efficacy or tolerability as reflected by its highest persistence and highest return rates.

\section{Funding: No funding sources Conflict of interest: None declared}

Ethical approval: The study was approved by the Institutional Ethics Committee 


\section{REFERENCES}

1. Chute CG, Panser LA, Girman CJ, Oesterling JE, Guess HA, Jacobsen SJ, et al. The prevalence of prostatism: a population-based survey of urinary symptoms. J Urol. 1993;150:85-9.

2. Konwar R, Chattopadhyay N, Bid HK. Genetic polymorphism and pathogenesis of benign prostatic hyperplasia. BJI Int. 2008;102:536-44.

3. Woo HH, Gillman MP, Gardiner R, Marshall V, Lynch WJ. A practical approach to the management of lower urinary tract symptoms among men. Med J Aust. 2011;195:34-9.

4. McConnell JD, Barry MJ, Bruskewitz RC. Benign prostatic hyperplasia: diagnosis and treatment. Agency for Health Care Policy and Research. Clin Pract Guidel Quick Ref Guide Clin. 1994;8:1-17.

5. American Urological Association. American Urological Association guideline: management of benign prostatic hyperplasia (BPH). Linthicum (MD): American Urological Association Education and Research, Inc.; 2010. Available at: www.auanet.org/guidelines/benign-prostatic-

hyperplasia- (2010-reviewed-and-validity-confirmed2014). Accessed on 25 July 2019.

6. Chughtai B, Forde JC, Thomas DM, Laor L, Hossack $\mathrm{T}$, Woo $\mathrm{HH}$ et al. Benign prostatic hyperplasia. Nature Reviews Disease Primers. 2016;2:16031.

7. Lepor H. Alpha Blockers for the Treatment of Benign Prostatic Hyperplasia. Rev Urol. 2007;9(4):181-90.

8. WHO Global Health Observatory (GHO) data. Available at: https://www.who.int/gho/mortality _burden_disease/life_tables/situation_trends_text/en/ Accessed 25 July 2019.

9. Emberton M, Zinner N, Michel MC, Gittelman M, Chung MK, Madersbacher S. Managing the progression of lower urinary tract symptoms/benign prostatic hyperplasia: therapeutic options for the man at risk. BJU Int. 2007;100:249-53.
10. Emberton M, Marberger M, de la Rosette J. Understanding patient and physician perceptions of benign prostatic hyperplasia in Europe: The Prostate Research on Behaviour and Education (PROBE) Survey. Int J Clin Pract. 2008;62(1):18-26.

11. Sabnis RB, Rajeev TP, Sinha S, Srivastava Aneesh, Mittal R. Survey by urological society of India (USI) to understand practice patterns in diagnosis and medical management of voiding LUTS secondary to benign prostatic obstruction (BPO). Int J Sci Res. 2019;8(5):18-21.

12. Tomašković I, Tomić $M$, Nikles S, Neretljak I, Miličić V. Croatian urologists'clinical practice and compliance with guidelines in the management of non-neurogenic male lower urinary tract symptoms. Acta Clin Croatica. 2015;54(4):453-7.

13. Wu N, Sun J, Yu P, Sun Z. Chinese Urologists' Views of Practice Patterns in the Diagnosis and Treatment of Benign Prostatic Hyperplasia: A Nationwide Survey. Int Neurourol J. 2012;16(4):1915.

14. Oh CY, Lee SH, Yoo SJ, Chung BH. Korean urologist's view of practice patterns in diagnosis and management of benign prostatic hyperplasia: a nationwide survey. Yonsei Med J. 2010;51(2):24852.

15. Moon HW, Yang JH, Choi JB, Bae WJ, Cho HJ, Hong SH, et al. Prescription pattern of alpha blockers for management of lower urinary tract symptoms/benign prostatic hyperplasia. Sci Rep. 2018;8:13223.

Cite this article as: Tomar V, Bhati BS, Datta B, Bellamkonda L, Prajapati HK, Chaturvedi A, et al. Prescription pattern of alpha blockers in the treatment of benign prostatic hyperplasia in India: a paper based survey. Int J Basic Clin Pharmacol 2020;9:348-52. 